\title{
Epithelial-mesenchymal transition (EMT) in kidney fibrosis: fact or fantasy?
}

\author{
Wilhelm Kriz, ${ }^{1}$ Brigitte Kaissling, ${ }^{2}$ and Michel Le Hir ${ }^{2}$ \\ 1Department of Anatomy and Developmental Biology, Medical Faculty Mannheim, University of Heidelberg, Mannheim, Germany. \\ 2Institute of Anatomy, University of Zurich, Zurich, Switzerland.
}

\begin{abstract}
Epithelial-mesenchymal transition (EMT) has become widely accepted as a mechanism by which injured renal tubular cells transform into mesenchymal cells that contribute to the development of fibrosis in chronic renal failure. However, an increasing number of studies raise doubts about the existence of this process in vivo. Herein, we review and summarize both sides of this debate, but it is our view that unequivocal evidence supporting EMT as an in vivo process in kidney fibrosis is lacking.
\end{abstract}

Decline in renal function in chronic renal failure is caused by a progressive loss of viable nephrons. Kidney diseases that progress to chronic renal failure most often start with problems in the glomerulus. From there, the disease process spreads to the tubulointerstitium, leading to the loss of the entire nephron and its replacement by a fibrotic scar. This is a focal process - meaning that individual nephrons are lost and replaced by fibrotic tissue on a local basis (1). There are many important questions open in this sequence: how glomerular disease transfers to the tubule has been controversial, as has the process by which injury from the tubule transfers to the interstitium, culminating in fibrosis (2). Among the mechanisms responsible for this final step, epithelial-mesenchymal transition (EMT) has become a popular hypothesis.

EMT is a process well known in ontogeny. In an early embryo, the epithelial layer (epiblast) gives rise to most, if not all, mesodermal structures of the body. Also, later in ontogeny, epithelial cells (e.g., cells of the somites) transform into mesenchymal cells. Thus, in ontogeny, mesenchymal cells emerge from epithelial cells. In recent reviews, this type of EMT has been classified as type 1 (3).

In pathological processes, EMT has been described to occur in carcinogenesis. In that realm, the dedifferentiation of cells with loss of epithelial and acquisition of mesenchymal features is also termed EMT (4) and has been recently classified as type 3 EMT (3).

Another type of pathological EMT (classified as type 2) has been proposed to occur in a parenchymal organ (liver, lung, or kidney) when specific cells are lost in the course of a disease and are replaced by fibrotic tissue. This hypothesis is highly controversial. It postulates that parenchymal cells (hepatocytes, alveolar cells, or renal tubular cells undergo transition into myofibroblasts, which then produce collagenous matrix contributing to organ fibrosis $(3,5-7)$.

We will discuss only type 2 EMT in the context of kidney fibrosis here, listing the pros and cons of the hypothesis that renal tubular cells, in the setting of parenchymal injury, undergo transition into mesenchymal cells, which participate in the development of fibrosis.

\section{The hypothesis}

Let us first consider this hypothesis in more detail. Renal tubular epithelia consist of a single layer of cells that are connected to each other by a girdle of intercellular junctions on the apical side

Conflict of interest: The authors have declared that no conflict of interest exists. Citation for this article: JClin Invest. 2011;121(2):468-474. doi:10.1172/JCI44595. and are attached to a tubular basement membrane (TBM) on the basal side. They have apical and basolateral cell membrane compartments, each with its own inventory of transporters and other membrane proteins. Mesenchymal cells (including fibroblasts and myofibroblasts) do not have this kind of polarity; instead they display cytoplasmic processes that make contacts - generally in a point-like manner - to other cells, capillaries, and tubules.

The transition of a tubular epithelial cell into a myofibroblast is thought to be initiated by cell injury. At an early stage of tubular degeneration, some cells will respond to stimuli of autocrine and/or paracrine origin by downregulating their epithelial and upregulating a mesenchymal genetic program. The new cells are thought to produce proteases that dissolve the TBM locally, allowing cells to move through the TBM and to settle in the peritubular interstitium. Here they promote fibrosis by producing type I collagenous matrix $(3,8)$.

Structural details of this process have so far not been elucidated either by transmission electron microscopy (TEM) or by light microscopy. The hypothesis has been derived exclusively from the observation that cells of injured tubules lose epithelial markers while they acquire mesenchymal marker proteins. Therefore, we first discuss the reliability and relevance of the markers that were generally used in this context.

\section{Markers of EMT}

The seminal work on EMT in kidney fibrosis was published in 1995 by Strutz and coworkers (9), in which FSP1 was introduced as a marker for cells undergoing EMT. FSP1 was described as a protein that, in primary cultures of murine renal cells, was expressed by fibroblasts but not by epithelial cells. Thus the authors coined the term "fibroblast-specific protein-1," although the protein was already known as S100A4 in the cancer literature. Strutz et al. (9) reported a low incidence of FSP1-positive cells in healthy kidneys, while in a model of renal fibrosis they observed a massive increase in their incidence in the interstitium and, more importantly, in tubular epithelia. Two conclusions were drawn from those observations. First, fibroblasts are scarce in the healthy kidney. Second, under pathological conditions, interstitial fibroblasts originate from tubular cells undergoing EMT. This work has stimulated widespread use of FSP1 antibodies for the identification of fibroblasts in order to detect EMT (10-14).

In light of our current knowledge, the use of FSP1/S100A4 antibodies as a specific marker for fibroblasts is not tenable. The interstitium of the healthy kidney contains an extensive network of 


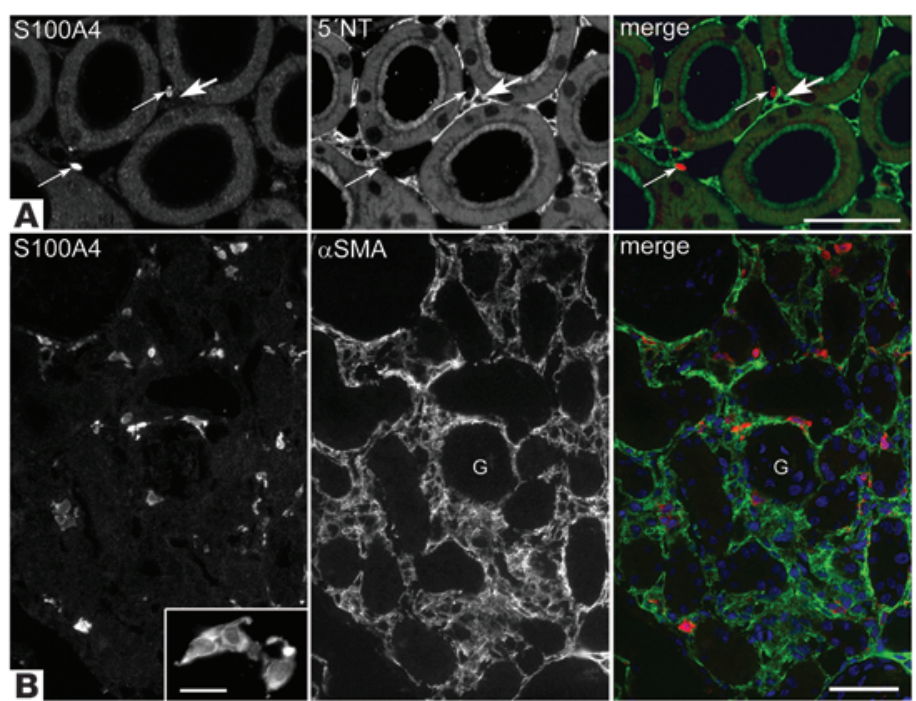

\section{Figure 1}

In the kidney, FSP1/S100A4 does not stain fibroblasts or myofibroblast. (A) Double immunofluorescence for S100A4 (red channel) and ecto-5'nucleotidase (5'NT, green channel) and (B) for S100A4 (red channel) and $\alpha$-SMA (green channel); nuclei in B are stained by DAPI. (A) 3-mm-thick cryostat section of renal cortex (rat); the thick arrow points to a $5^{\prime} \mathrm{NT}$-positive interstitial fibroblast; S100A4-positive cells (thin arrows) are seen inside capillaries. Scale bar: $\sim 10 \mathrm{~mm}$. Reproduced with permission from Histochemistry and Cell Biology (15). (B) Renal cortex after 4 days of ureteral obstruction (rat); no cellular colocalization of S100A4 and $\alpha$-SMA. G, glomerulus. Inset: S100A4-positive cells, showing strong cytoplasmic and weak nuclear staining; the shape of the cells is reminiscent of migrating lymphocytes. Scale bars: $\sim 100 \mathrm{~mm}$; inset, $\sim 10 \mathrm{~mm}$. Reproduced with permission from Histochemistry and Cell Biology (17). fibroblasts (Figure 1), which do not express FSP1/S100A4 $(15,16)$; also, myofibroblasts do not express FSP1/S100A4 (17). The FSP1/ S100A4-positive cells in the renal interstitium belong rather to the immune system $(14,15,18-20)$. In addition, the endothelial cells of some renal microvessels express FSP1/S100A4 $(15,19)$. These findings are in agreement with the detection of S100A4 in inflammatory cells and in some endothelia in humans (21).

Similarly, another marker used to indicate EMT, vimentin, cannot be taken as an unequivocal marker for mesenchymal cells. In rat and human material, it was shown (22) that vimentin is very commonly expressed in injured tubular cells not only during tubular degeneration in chronic diseases, but also during regeneration in acute tubular injury. Later studies $(15,23,24)$ showed that the expression of vimentin in injured tubular cells displayed a clear basolateral, thus an epithelial, pattern. In a model of transiently injured tubular cells (23), epithelial repair took place by local proliferation of surviving cells. Vimentin expression was maintained at a high level until day 5 after ischemia; thereafter, it decreased concurrently with recovery. This observation supports the conclusion by Gröne and colleagues (22) that vimentin may be regarded as an indicator of tubular regenerating activity.

$\alpha$-SMA is the characteristic actin isoform found in vascular smooth muscle cells, pericytes, and myofibroblasts $(25,26)$. Proximal tubular cells contain a cytoskeleton with prominent F-actin microfilaments that do not stain with antibodies against $\alpha$-SMA. In some studies of renal disease models with fibrosis expression, $\alpha$-SMA has been found in tubular cells, and that finding was considered as evidence for EMT (27-29). However, in most other studies $\alpha$-SMA was exclusively found in interstitial cells around injured tubules, whereas the tubules themselves remained $\alpha$-SMA-negative $(15,17,19,30-33)$. The same observation was made in biopsies of patients with nephritic syndrome (34).

Since direct detection of collagen synthesis by immunolabeling has not yet been achieved, proteins that participate in its synthesis, HSP 47 (a collagen-specific molecular chaperone) and prolyl-4-hydroxylase, have been used as surrogate markers. Expression of HSP47 in injured tubules has been taken as evidence for synthesis of collagen by tubular cells and thus for EMT $(10,11)$. However, HSP47 is not specific for collagen type I (35) but is equally involved in collagen type IV synthesis, and thus in the synthesis of TBM collagen.
The loss of proteins that are typically found only in epithelia has also been used to indicate EMT. Most frequently, the loss of keratin, $\beta$-catenin, E-cadherin, and the kidney-specific cadherin 16 (36) has been invoked in this respect (37). When tubules undergo decomposition, the cells progressively lose their intercellular junctional contacts, and also their contacts with the basement membrane. Thus, it is not surprising that they lose some of the junctional proteins. This, however, by no means constitutes evidence of a mesenchymal phenotype.

In summary, a variety of markers were used to establish evidence for EMT. Since none of the markers is unequivocally indicative of EMT, it is our view that marker expression data are not sufficient to prove EMT.

\section{Experimental studies supporting EMT}

The crucial study supporting EMT in kidney fibrosis is a cell fate tracing study published by Iwano and coworkers in 2002 (10). The authors used the unilateral ureteral obstruction (UUO) model to induce kidney fibrosis in mice. They permanently labeled tubular cells with LacZ using the $\gamma \mathrm{GT}$ promoter and showed that within a degenerating tubule, epithelial cells may acquire FSP1 positivity and that cells positive for both LacZ and FSP1 appeared to have settled in the fibrotic tissue surrounding the tubules. Moreover, colocalization of HSP47 in LacZ-positive cells located in the peritubular interstitium was taken as evidence that these cells were derived from epithelial cells and that they participated in the production of fibrous collagens.

It is our opinion that this study contains serious flaws. Later studies $(33,38)$ showed that the immunofluorescent labeling of $\mathrm{LacZ}$ is prone to artefacts, which may account for some of the following inconsistencies in the crucial Figure 2 of the study by Iwano and coworkers (10), reproduced here as Figure 2. First, $\gamma$ GT is a brush border protein, expressed specifically in the proximal tubules. However, all tubules in the control (Figure 2A) appear equally labeled, although two profiles (both at 7:00 from the glomerulus, one of them partially covered by "fibroblasts") are definitely not proximal. Second, the LacZ labeling is homogeneous in all tubular cells. However, insertion of a transgene using Cre recombinase usually yields variable levels of expression. Third, the merged pictures showing the colocalization of LacZ and FSP1 or HSP47 


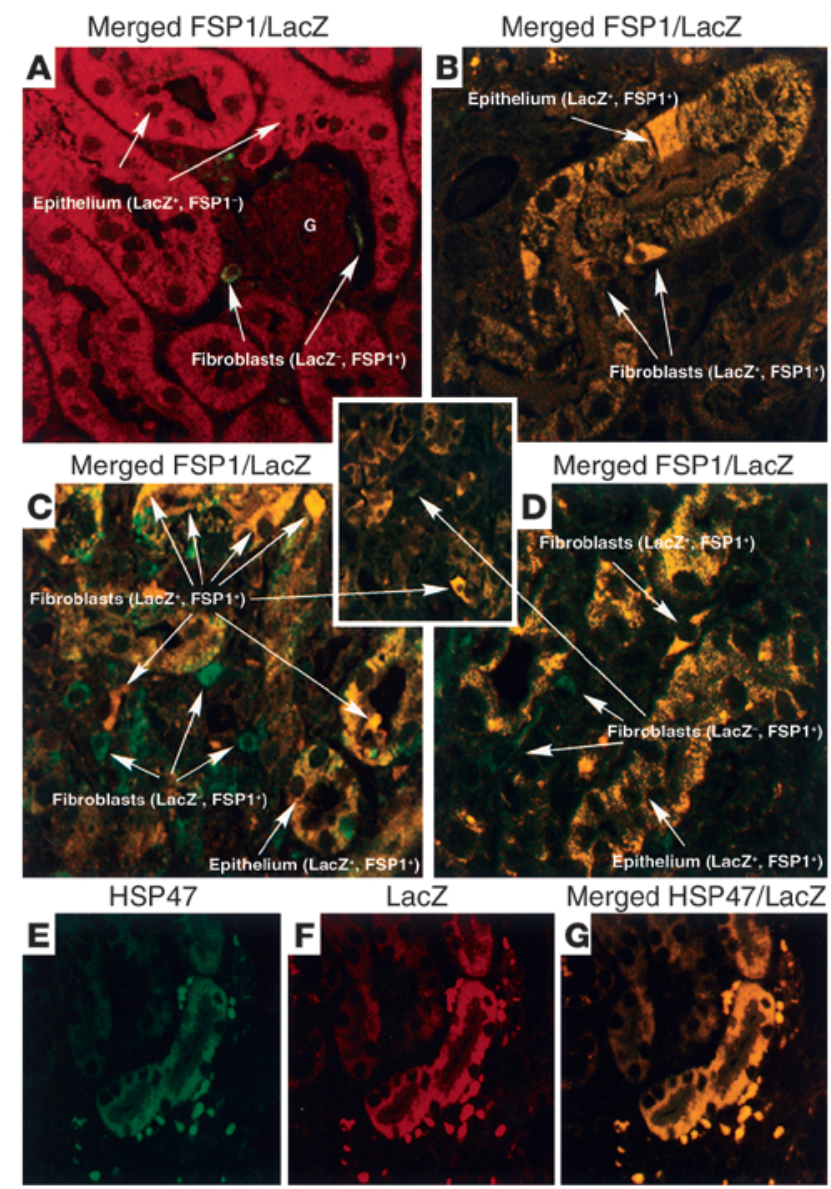

Figure 2

Reproduction of Figure 2 from the article by Iwano et al. (10).

after UUO exhibit a complete overlap in location and strength, quite obvious in panels E, F, and G, but also in panels B and D. This is possible only under the very unlikely condition that the levels of expression of the two proteins are in a constant ratio at all sites where coexpression takes place. Fourth, the FSP1 antibody is not suitable to identify fibroblasts, as is illustrated in panel A, where there are three green-labeled $\left(\mathrm{FSP} 1^{+}\right)$structures, two of them designated as fibroblasts. However, on the basis of structural studies (39), the area shown in panel A contains more fibroblasts. In addition, the labeled cell at 8:00 from the glomerulus is a round cell hardly compatible with a fibroblast. Last, in panel C, seven arrows point to $\mathrm{LacZ}^{+} \mathrm{FSP} 1^{+}$structures, i.e., cells that have undergone EMT, suggesting a high frequency of such an event. This is hardly compatible with the fact that cells in the process of EMT have so far not been found in structural studies.

The study by Iwano and coworkers (10) stimulated widespread activity in the field. Numerous studies were undertaken aiming at elucidating details of this new mechanism. Most of them were cell culture studies $(12,40-45)$ that clearly showed that renal tubular epithelial cells when exposed to profibrotic cytokines (especially TGF- $\beta 1$ ) lose cell polarity, downregulate epithelial markers (E-cadherin, $\beta$-catenin, zonula occludens protein 1 ), acquire mesenchymal features (become spindle-shaped and mobile, produce MMP-2), and express mesenchymal markers (vimentin, $\alpha$-SMA). This pat- tern of changes was taken to be a common signature for EMT and was proposed to be valid as indirect evidence of EMT in vivo $(5,46)$. In a recent review supporting the EMT hypothesis (3), it was conceded that the phenotypic alterations in vitro characterize a "partial EMT," lacking the final transition to a "fully fibroblastic phenotype." These studies do not contribute any relevant evidence for a role of EMT in renal fibrosis, which clearly requires a "fully fibroblastic phenotype.”

Among the experimental in vivo studies supporting EMT, there were a few that used routine techniques of light and electron microscopy and claimed to show morphological alterations in proof of $\operatorname{EMT}(11,36,40)$; however, the quality of the morphological data was not sufficient for that purpose. Neither a cell with characteristics of a fibroblast within the TBM, nor a cell crossing the TBM, nor gaps in the TBM were properly documented.

In a second category of pro-EMT articles, EMT was assumed as an established fact, and the study was designed to uncover details of this mechanism in the context of renal fibrosis. Data that were compatible with EMT were considered as evidence for EMT. This type of circular reasoning has been applied in several studies manipulating the activity of TGF- $\beta$ in models of renal fibrosis $(41,47,48)$. According to these studies, the reduction of fibrosis in response to suppression of the TGF- $\beta$ pathway reflected a blocking effect on EMT. Other possible effects were not considered.

Similar reasoning was found in the study that used a tamoxifen-inducible Snail1 transgenic mouse model (36) that expressed significant levels of the protein in the kidney. The Snail family of transcription factors induces EMT in vitro, shown in a mouse mammary gland-derived epithelial cell line (NMuMG) (36). Induction of Snail1 in the above in vivo model resulted in an increase in interstitial fibrous collagen. This was interpreted only in the context of EMT, although the authors did not provide any specific evidence of EMT. In a recent study (49), activation of the Notch pathway (involving the Snail transcription factors) was shown to induce upregulation of mesenchymal markers in vitro but not in vivo.

Venkov and coworkers (12) showed in a proximal tubule epithelial cell line that the transcription factor CBF-A led to downregulation of epithelial-specific proteins and upregulation of mesenchymal-specific proteins including FSP1. In an attempt to investigate a possible role of CFB-A in vivo, the authors examined the localization of CFB-A and the expression of FSP1 in the UUO model in FSP1/GFP mice. Their data are confusing; we cannot retrace how the authors came to the conclusion that these pictures show FSP1-positive cells that were derived from CBF-A-stimulated tubular cells and settled in the interstitium (Figure 3). In the text the authors simply state, "This series of images suggests that the nuclei of many tubular cells in the UUO kidney are enriched for CBF-A and potentially positioned for an EMT event." Thus, the authors do not really claim to show EMT in the UUO model.

In summary, none of these studies contain robust evidence for a contribution of EMT to kidney fibrosis. None of them met the crucial requirement of showing that in vivo, tubular cells/tubule-derived cells were capable of producing type I collagenous matrix, and none of them presented evidence for a migration of cells across the TBM. The cell culture studies did uncover a certain pattern of de-differentiation of tubular epithelial cells, including the upregulation of mesenchymal proteins that would be relevant to the EMT hypothesis. However, this pattern does not include evidence for the decisive final steps to 


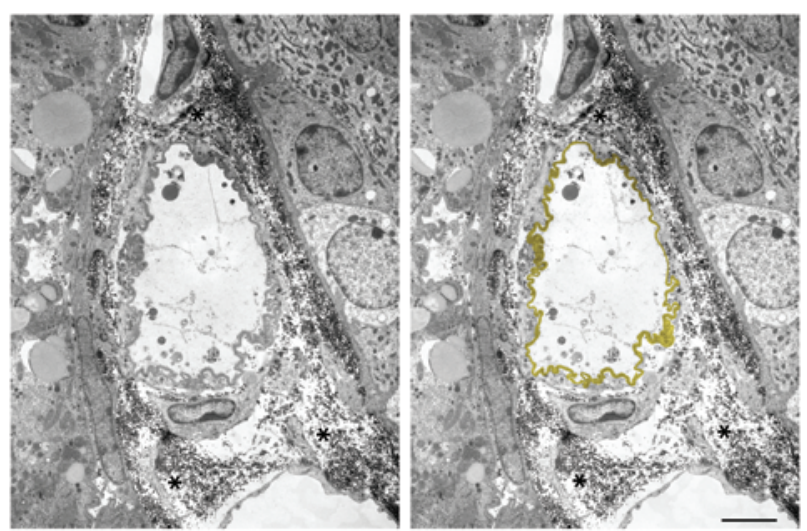

Figure 3

Empty profile of a degenerating tubule. TEM of tubular remnant from a transgenic mouse with tubular overexpression of TGF- $\beta 1$ (50). This profile is devoid of any cells but is maintained as an entity by the presence of a continuous TBM (highlighted in yellow). Note that there is massive deposition of fibrous collagen (asterisks) around this tubular remnant, but there is not a single fiber inside. Scale bar: $5 \mu \mathrm{m}$.

a complete EMT, i.e., phenotype switch and ability to produce fibrous matrix; so we are left with only a "partial EMT" $(3,8)$ whose pathological relevance remains unclear.

\section{Studies that did not find evidence for a contribution of EMT to renal fibrosis}

Cell fate tracing studies. As mentioned above, the cell fate tracing study in the UUO model by Iwano and coworkers (10) has been widely accepted as definitive evidence of EMT in the kidney; however, the results of this article have so far not been confirmed by any other study. In contrast, two different groups using fate-tracing techniques in the UUO model did not find any evidence for a contribution of EMT to renal fibrosis $(19,33)$.

Humphreys and coworkers used three Cre/Lox transgenic lines in which specific populations of renal cells were genetically labeled (33). First, using the Six 2 promoter, all tubular cells derived from the cap mesenchyme (nephron anlage); and second, using the Hoxb7 promoter, all tubular cells derived from the ureteral bud were labeled. Third, as a positive control, all renal mesenchyme-derived cells were labeled (using the transcription factor FoxD1). In all three transgenics, two different labels were used, LacZ and Z/Red. After UUO, in the first two models no cells were found in the peritubular interstitium that were positive for the epithelial markers; thus, no cells were found that settled in the peritubular interstitium. In the third transgenic line, labeled myofibroblasts were easily detected in the peritubular interstitium, proving that resident mesenchymal cells may transform into myofibroblasts. Moreover, treatment with TGF- $\beta 1$ of cultured proximal tubule cells derived from the Six 2 GC; Z/Red mice consistently led to the induction of mesenchymal markers. Identical results were obtained in an ischemia-reperfusion injury model. Taken together, the findings of this rigorous study clearly show that tubular cells may acquire mesenchymal markers in vitro. However, in vivo, tubular cells did not transform into any cells contributing to fibrosis.

In another recent study, the authors used the Ksp-cadherin promoter to label all renal tubular cells with enhanced yellow fluorescence protein (EYFP) as a permanent marker (19). After UUO, cells positive for EYFP were confined within the TBM, were not found in the interstitium, and did not express markers of fibroblasts or myofibroblasts.

A third cell fate tracing study addressed this question in a transgenic mouse model, in which renal fibrosis was induced by TGF- $\beta 1$ overexpression in renal tubules (50). In this study, Pax8-rtTA mice (51) were used to drive the simultaneous expression of TGF- $\beta 1$ and Cre recombinase in renal tubular epithelial cells. Whereas TGF- $\beta 1$ expression triggered tubular damage and renal fibrosis, Cre recombinase expression led to recombination at the Rosa26-lacZ locus (the fourth transgene employed in this model), thereby irreversibly labeling the tubular epithelial cells with $\beta$-galactosidase activity. TGF- $\beta 1$ is considered as the principal cytokine that induces EMT (52). However, even under these seemingly favorable conditions for EMT, labeled epithelial cells were never found in an interstitial position within fibrotic areas.

Furthermore, in two animal models of renal fibrosis, UUO (19) and habu venom injury (32), tubular epithelia were labeled with Texas red-dextran (TR-dextran) and tracked. In both studies, TR-dextran-retaining cells were found in cells of atrophic tubules but remained excluded from the peritubular interstitium.

Taken together, five cell fate and/or cell-labeling studies from different groups did not find any evidence that renal tubular cells transform into mesenchymal cells, more specifically into fibroblasts or myofibroblasts.

Structural studies. One of the most attractive features of the EMT hypothesis is the idea that injured cells themselves contribute to fibrosis and thus to repair through scarring. In contrast to the liver, in the kidney these two processes - cell injury and fibrosis development - are spatially separated from each other: cell injury occurs within, fibrosis development outside the tubular compartment. Therefore, the transition of tubular epithelial cells into fibroblasts/myofibroblasts by EMT means both a fundamental change in cell shape, which is expected to happen inside the TBM, and a change in cell location, i.e., these cells have to cross the TBM and to settle in the interstitium. Under this scenario, we would expect to find cells with a mesenchymal morphology both inside the TBM and in transit to the outside crossing the TBM. Such events, if not extremely rare and thus of questionable significance, should be easily documented in structural studies by TEM. So far, no pictures are found in the literature showing such cells.

As has been shown in previous studies $(1,53-55)$, the process of tubular degeneration occurs in a certain sequence. First, cells simplify their structural elaborations, losing their surface differentia-

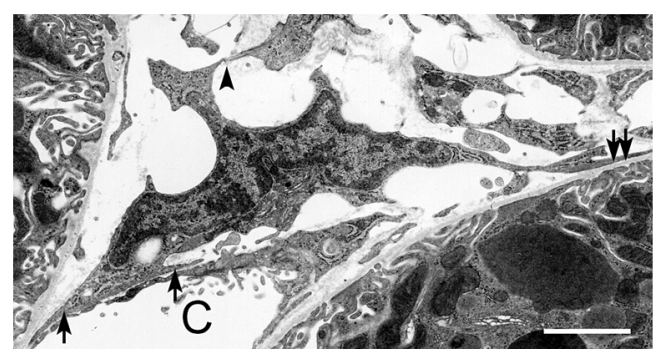

\section{Figure 4}

Renal fibroblast. TEM of a fibroblast in the renal cortical interstitium of a healthy rat. From the cell body, numerous processes emerge that make contacts with a capillary (C, arrows), a tubule (double arrow), and another fibroblast (arrowhead). Scale bar: $1 \mu \mathrm{m}$. Reproduced with permission from Histochemistry and Cell Biology (16). 


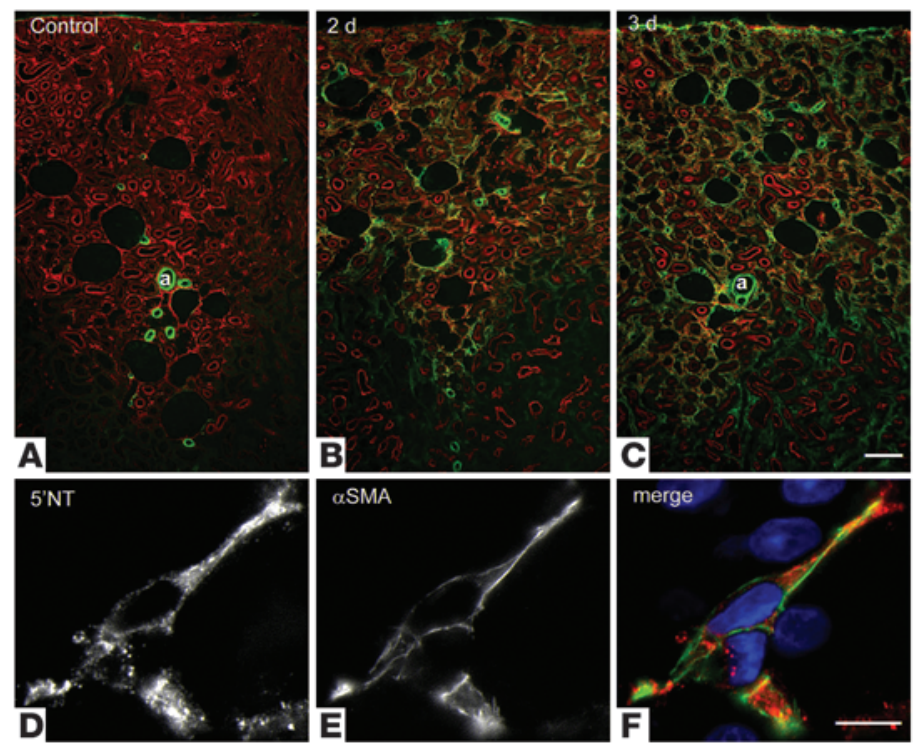

\section{Figure 5}

Fibroblasts are the origin of myofibroblasts. Rat renal cortex in sham-operated $(\mathbf{A})$ and in ureter-ligated kidneys of rats ( $\mathbf{B}$ and C) (3- $\mu \mathrm{m}$ cryostat sections; red: $5^{\prime} \mathrm{NT}$, green: $\alpha-S M A$, blue: cell nuclei). In controls (A), the interstitium and the brush border of proximal tubules are strongly labeled by $5^{\prime} \mathrm{NT} ; \alpha$-SMA labels exclusively arterial vessels (A). After 2 days of ureter ligature (B), interstitial $5^{\prime} \mathrm{NT}$ staining decreases, whereas $\alpha$-SMA appears and becomes increasingly prominent throughout the cortex after 3 days (C). (D-F) Interstitial fibroblast in ureter-ligated kidney after 2 days. The weakly expressed $5^{\prime} \mathrm{NT}$ is distributed in a granular manner over the plasma membrane and the cytoplasm; $\alpha$-SMA is apparent along the plasma membrane and in the cellular processes. Scale bars: $100 \mu \mathrm{m}(\mathbf{A}-\mathbf{C}), 10 \mu \mathrm{m}(\mathbf{D}-\mathbf{F})$. Reproduced with permission from Histochemistry and Cell Biology (17).

tion, but maintaining cell polarity even after collapse of the lumen. The process of decomposition generally starts with the accumulation of lysosomal elements indicating autophagy $(19,50)$. In this stage, the degenerating cells generally lose their fixation to the TBM, frequently forming strands of dying cells. There is no noticeable invasion of the tubule by inflammatory cells; thus, autophagy and fratricide are the most likely mechanisms accounting for the disappearance of the tubular cells.

It is important to remember that during all these stages of degeneration, the integrity of the TBM - even if it becomes collapsed and highly folded - is preserved (Figure 3). This survival of the TBM represents the crucial prerequisite for the high regeneration capacity of the tubular epithelium in acute renal failure.

Over the years, we have studied tubular degeneration associated with fibrosis in various rat and mouse models. Since at the time of most of these studies EMT was not yet in discussion, we did not look for it. However, we described the specific pattern of tubular degeneration eventually leading to almost cell-free remnants of intact, frequently thickened TBM $(54,56,57)$.

In two recent studies $(50,58)$, we deliberately searched in a large number of electron micrographs for cells crossing the TBM from inside the tubular compartment to outside. We did not find such cells. Similar efforts were undertaken in an model of acute renal failure (59); also, in this study cells crossing the TBM were not encountered.

These data only show that any cells in transit were not found; they do not prove that such cells do not exist. Yet after 15 years of EMT research, cells in transit from inside an injured tubule through the TBM into the peritubular space have only been shown in schematics. Not a single picture of a cell in transit has ever been published, much less any regular structural evidence that might give significance to this mechanism.

In the model of TGF- $\beta$-induced renal fibrosis (50), we also addressed the question of whether tubular cells underwent EMT and produced collagen still within the tubular compartment. From this study, Figure 3 shows almost cell-free tubular remnants with massive deposition of fibrous collagen in their surroundings, but without a single fiber inside. Cells with a mesenchymal morphology were never encountered inside the TBM.
In summary, though EMT describes a process with manifold structural implications, no structural evidence has so far been presented that supports this hypothesis.

\section{Studies showing that the bulk of myofibroblasts originate from fibroblasts}

Until the mid-1990s, nobody questioned that organ fibrosis develops from proliferation of local fibroblasts and their conversion into myofibroblasts, which proliferate and produce the fibrous intercellular matrix (60-64). Since that time, however, two other sources of myofibroblasts came into discussion, bone marrow-derived fibrocytes and tubular cells undergoing EMT. The contribution of bone marrow-derived fibrocytes is generally believed to be small (65-67).

Nevertheless, there is a widespread resistance to the idea that local resident interstitial cells are the major, if not the only, source for the fibrosis-producing myofibroblasts. These interstitial cells are traditionally termed fibroblasts. They are the ubiquitous cells that perform a scaffolding function interconnecting the various organ-specific substructures - in the kidney, these are the nephrons and the supplying capillaries. They perform this function by focal contacts of their cytoplasmic processes with neighboring fibroblasts as well as with capillaries and tubules (refs. 39, 68, and Figure 4). In addition, they produce the fibrous matrix, which serves together with these cells as a common scaffold throughout the renal parenchyma.

Interstitial fibroblasts in the kidney cortex can be unequivocally detected by electron microscopy and fluorescence microscopy by their strong expression of ecto-5'-nucleotidase (CD73) $(16,33,39$, $68,69)$. Some unique ultrastructural features, such as the abundance of rough ER (RER) and F-actin filaments and an association with collagen fibrils, make identification in the electron microscope easy.

In the UUO model, Picard and coworkers (17) investigated the alterations of fibroblasts upon conversion into myofibroblasts. Relevant changes were seen already on the first day following ligature consisting of the appearance of bundles of actin microfilaments and of abundant cisterns of RER. Double immunofluorescence revealed the gradual expression of $\alpha$-SMA exclusively in ecto- $5^{\prime}$ nucleotidase-positive cells. Thus, the conversion of a fibroblast 
into a myofibroblast was documented in a continuous sequence of steps (Figure 5). A corresponding continuous sequence was also documented after uranyl acetate-induced acute renal failure by transmission and scanning electron microscopy (59).

There are several other recent in vivo studies showing that the myofibroblasts emerge from proliferation of local interstitial cells. Lin and coworkers (65) used a coll1a1-GFP transgenic mouse line to identify pericytes/fibroblasts as the major contributors to the myofibroblast population in UUO. In a cell fate study, using transgenic mice in which LacZ was expressed specifically in pericytes/ fibroblasts, Humphreys and coworkers (33) found that the totality of $\alpha$-SMA-expressing cells in the interstitium originated from pericytes/fibroblasts both in UUO and after ischemia-reperfusion injury. A further study (32) showed similar results in an accelerated model of angiotensin II-induced renal fibrosis.

These studies certainly do not rule out a source of myofibroblasts from other cell populations, but they show that the transition of fibroblasts into myofibroblasts occurs on a large scale and may occur very early after injury, long before tubular degeneration.

A final point remains to be discussed: the controversy over whether these resident interstitial cells should be termed fibroblasts or pericytes. The fact that renal interstitial fibroblasts have frequent contacts with capillaries (Figure 4) led some authors to identify these cells as pericytes $(33,65)$. However, the same cells have frequent contacts with tubules and other interstitial cells. In total, they are identified in the renal cortex by the expression of ecto-5'-nucleotidase (CD73) $(16,33)$ as well as of PDGFR $\beta$ (65). If we term these interstitial cells pericytes, the peritubular interstitium of the kidney would be devoid of any fibroblasts.

Fibroblasts are an exceptionally versatile cell type with strong organ-specific elaborations, but the common function in every organ is to provide the scaffold that lends mechanical support to tissue architecture. From this point of view, it makes sense that they are responsible for fibrosis.

\section{Conclusion}

In summary, we feel there are no solid data supporting EMT as an in vivo process in kidney fibrosis. We are not aware of any study showing transdifferentiation of a tubular epithelial cell into a cell capable of synthesizing fibrous matrix, nor one presenting a tubular cell in the process of transit across the TBM. The only cell fate tracing study that supports EMT awaits confirmation, while other fate tracing studies cast strong doubts on the existence of EMT. It is hard to understand why EMT has become so deeply ingrained into fibrosis research. Similar doubts about a role of EMT in organ fibrosis have recently been raised concerning the liver $(38,70)$.

On the other side, there is ample evidence from various animal models and human pathology that kidney fibrosis results from the proliferation of resident fibroblasts transforming into myofibroblasts. There are strong reasons to focus on this cell population in studies of renal fibrosis.

\section{Acknowledgments}

We would like to thank Benjamin Humphreys for helpful discussions.

Address correspondence to: Wilhelm Kriz, Medizinische Fakultät Mannheim der Universität Heidelberg, Abteilung: Anatomie und Entwicklungsbiologie, Ludolf-Krehl-Str. 13-17, Tridomus C, Ebene 6, D68167 Mannheim, Germany. Phone: 49.0.621.383.9941; Fax: 49.0.621.383.9949; E-mail: wilhelm.kriz@urz.uni-heidelberg.de.
1. Kriz W, Le Hir M. Pathways to nephron loss starting from glomerular diseases - insights from animal models. Kidney Int. 2005;67(2):404-419.

2. Cook H. The origin of renal fibroblasts and progression of kidney disease. Am J Pathol. 2010; 176(1):22-24.

3. Kalluri R, Weinberg R. The basic of epithelial-mesenchymal transition. J Clin Invest. 2009; 119(6):1420-1428.

4. Thompson E, Newgreen D. Carcinoma invasion and metastasis: a role for epithelial-mesenchymal transition? Cancer Res. 2005;65(14):5991-5995.

5. Kalluri R, Neilson E. Epithelial-mesenchymal transition and its implications for fibrosis. J Clin Invest. 2003;112(12):1776-1784.

6. Zeisberg E, et al. Fibroblasts derive from hepatocytes in liver fibrosis via epithelial to mesenchymal transition. J Biol Chem. 2007;282(32):23337-23347.

7. Thiery J, Acloque H, Huang R, Nieto M. Epithelialmesenchymal transitions in development and disease. Cell. 2009;139(5):871-890.

8. Zeisberg M, Neilson E. Mechanisms of tubulointerstitial fibrosis. J Am Soc Nephrol. 2010; 21(11):1819-1834.

9. Strutz F, et al. Identification and characterization of a fibroblast marker: FSP1. J Cell Biol. 1995; 130(2):393-405.

10. Iwano M, Plieth D, Danoff T, Xue C, Okada H, Neilson E. Evidence that fibroblasts derive from epithelium during tissue fibrosis. J Clin Invest. 2002; 110(3):341-350.

11. Cheng S, Pollock A, Mahimkar R, Olson J, Lovett D. Matrix metalloproteinase 2 and basement membrane integrity: a unifying mechanism for progressive renal injury. FASEB J. 2006;20(11):1898-1900.

12. Venkov C, et al. A proximal activator of transcription in epithelial-mesenchymal transition. J Clin
Invest. 2007;117(2):482-491.

13. Zeisberg E, et al. BMP-7 counteracts TGF- $\beta 1$ induced epithelial-to-mesenchymal transition and reverses chronic renal injury. Nat Med. 2003;9(7):964-968.

14. Inoue T, Plieth D, Venkov C, Xu C, Neilson E. Antibodies against macrophages that overlap in specificity with fibroblasts. Kidney Int. 2005;67(6):2488-2493.

15. Le Hir M, Hegyi I, Cueni-Loffing D, Loffing J, Kaissling B. Characterization of renal interstitial fibroblast-specific protein 1/S100A4-positive cells in healthy and inflamed rodent kidneys. Histochem Cell Biol. 2005;123(4-5):335-346.

16. Kaissling B, Le Hir M. The renal cortical interstitium: morphological and functional aspects. Histochem Cell Biol. 2008;130(2):247-262.

17. Picard N, Baum O, Vogetseder A, Kaissling B, Le Hir M. Origin of renal myofibroblasts in the model of unilateral ureter obstruction in the rat. Histochem Cell Biol. 2008;130(1):141-155.

18. Rossini $\mathrm{M}$, et al. Immunolocalization of fibroblast growth factor-1 (FGF-1), its receptor (FGFR-1), and fibroblast-specific protein-1 (FSP-1) in inflammatory renal disease. Kidney Int. 2005;68(6):2621-2628.

19. Li L, Zepeda-Orozco D, Black R, Lin F. Autophagy is a component of epithelial cell fate in obstructive uropathy. Am J Pathol. 2010;176(4):1767-1778.

20. Burns W, Kantharidis P, Thomas M. The role of tubular epithelial-mesenchymal transition in progressive kidney disease. Cells Tissues Organs. 2007; 185(1-3):222-231.

21. Boye K, Maelandsmo G. S100A4 and metastasis: a small actor playing many roles. Am J Pathol. 2010; 176(2):528-535.

22. Gröne H, Weber K, Gröne E, Helmchen U, Osborne M. Coexpression of Keratin and Vimentin in damaged and regenerating tubular epithelia of the kidney.
Am J Pathol. 1987;129(1):1-8.

23. Witzgall R, Brown D, Schwarz C, Bonventre J. Localization of proliferating cell nuclear antigen, vimentin, c-Fos, and clustrin in the post-ischemic kidney. J Clin Invest. 1994;93(5):2175-2188.

24. Zhu M, Broe M, Nouwen E. Vimentin expression and distal tubular damage in the rat kidney. Exp Nephrol. 1996;4(3):172-183.

25. Gabbiani G, et al. Vascular smooth muscle cells differ from other smooth muscle cells: predominance of vimentin filaments and a specific $\alpha$-type actin. Proc Natl Acad Sci US A. 1981;78(1):298-302.

26 . Skalli $O$, et al. $\alpha$-smooth muscle actin, a differentiation marker of smooth muscle cells, is present in microfilamentous bundles of pericytes. J Histochem Cytochem. 1989;37(3):315-321.

27. Ng Y, et al. Tubular epithelial-myofibroblast transdifferentiation in progressive tubulointerstitial fibrosis in 5/6 nephrectomized rats. Kidney Int. 1998;54(3):864-876.

28 . Jinde $\mathrm{K}$, et al. Tubular phenotypic change in progressive tubulointerstitial fibrosis in human glomerulonephritis. Am J Kidney Dis. 2001;38(4):761-769.

29. Liu Y. Epithelial to mesenchymal transition in renal fibrogenesis: pathologic significance, molecular mechanism, and therapeutic intervention. J Am Soc Nephrol. 2004;15(1):1-12.

30. Kimura M, Asano M, Abe K, Miyazaki M, Suzuki $\mathrm{T}$, Hishida A. Role of atrophic changes in proximal tubular cells in the peritubular deposition of type IV collagen in a rat renal ablation model. Nephrol Dial Transplant. 2005;20(8):1559-1565.

31. Loffing J, et al. Thiazide treatment of rats provokes apoptosis in distal tubule cells. Kidney Int. 1996;50(4):1180-1190.

32. Faulkner J, Szcykalski L, Springer F, Barnes J. Origin of interstitial fibroblasts in an accelerated 
model of Angiotensin II-induced renal fibrosis. Am J Pathol. 2005;167(5):1193-1205.

33. Humphreys B, et al. Fate tracing reveals the pericyte and not epithelial origin of myofibroblasts in kidney fibrosis. Am J Pathol. 2010;176(1):85-97.

34. Kuusniemi A, Lapatto R, Holmberg C, Karikoski R, Rapola J, Jalanko H. Kidneys with heavy proteinuria show fibrosis, inflammation, and oxidative stress, but no tubular phenotypic change. Kidney Int. 2005;68(1):121-132.

35. Nagata K. Expression and function of heat shock protein 47: a collagen-specific molecular chaperone in the endoplasmic reticulum. Matrix Biol. 1998;16(7):379-386.

36. Boutet A, de Frutos C, Maxwell P, Mayol M, Romero J, Nieto M. Snail activation disrupts tissue homeostasis and induces fibrosis in the adult kidney. EMBO J. 2006;25(23):5603-5613.

37. Aresu L, et al. Dog as model for down-expression of E-cadherin and beta-catenin in tubular epithelial cells in renal fibrosis. Virchows Arch. 2008 453(6):617-625.

38. Taura K, et al. Hepatocytes do not undergo epithelial-mesenchymal transition in liver fibrosis in mice. Hepatology. 2010;51(3):1027-1036.

39. Kaissling B, Le Hir M. Characterization and distribution of interstitial cell types in the renal cortex of rat. Kidney Int. 1994;45(3):709-720.

40. Cheng S, Lovett D. Gelatinase A (MMP-2) is necessary and sufficient for renal tubular cell epithelial-mesenchymal transformation. Am J Pathol. 2003;162(6):1937-1949.

41. Zeisberg E, et al. BMP-7 counteracts TGF- $\beta 1$ induced epithelial-to-mesenchymal transition and reverses chronic renal injury. Nat Med. 2003; 9(7):964-968.

42. Slattery C, Campbell E, McMorrow T, Ryan M. Cyclosporine A-induced renal fibrosis. A role for epithelial-mesenchymal-transition. Am J Pathol. 2005;167(2):395-407.

43. McMorrow T, Gaffney M, Slattery C, Campbell C, Ryan A. Cyclosporine A induced epithelialmesenchymal transition in human renal proximal tubular epithelial cells. Nephrol Dial Transplant. 2005; 20(10):2215-2225

44. Ivanova L, Butt M, Matsell D. Mesenchymal transition in kidney collecting duct epithelial cells. Am J Physiol Renal Physiol. 2008;294(5):F1238-F1248.

45. Qi W, et al. Integrated actions of transforming growth factor- $\beta 1$ and connective tissue growth factor in renal fibrosis. Am J Physiol Renal Physiol. 2005;288(4):F800-F809.

46. Zeisberg E, Neilson E. Biomarkers for epithelial-mesenchymal transitions. J Clin Invest. 2009; 119(6):1429-1437.

47. Sato M, Muragaki Y, Saika S, Roberts A, Ooshima A. Targeted disruption of TGF- $\beta 1 /$ Smad 3 signaling protects against renal tubulointerstitial fibrosis induced by unilateral obstruction. J Clin Invest. 2003 112(10):1486-1494

48. Li Y, Yang J, Dai C, Wu C, Liu Y. Role for integrin-linked kinase in mediating tubular epithelial to mesenchymal transition and renal interstitial fibrogenesis. J Clin Invest. 2003;112(4):503-516.

49. Bielesz B, et al. Epithelial Notch signaling regulates interstitial fibrosis development in the kidneys of mice and humans. J Clin Invest. 2010; 120(11):4040-4054.

50 . Koesters R, et al. Tubular overexpression of transforming growth factor- $\beta 1$ induces autophagy and fibrosis but not mesenchymal transition of renal epithelial cells. Am J Pathol. 2010;177(2):632-643.

51. Traykova-Brauch $\mathrm{M}$, et al. An efficient and versatile system for acute and chronic modulation of renal tubular function in transgenic mice. Nat Med. 2008;14(9):979-984.

52. Zeisberg E, Kalluri R. The role of epithelial-tomesenchymal transition in renal fibrosis. J Mol Med. 2004;82(3):175-181.

53. Besse-Eschmann V, Le Hir M, Endlich N, Endlich $\mathrm{K}$. Alteration of podocytes in a murine model of crescentic glomerulonephritis. Histochem Cell Biol. 2004;122(2):139-149.

54. Kriz W, Hosser H, Haehnel B, Gretz N, Provoost A. From segmental glomerulosclerosis to total nephron degeneration and interstitial fibrosis: a histopathological study in rat models and human glomerulopathies. Nephrol Dial Transplant. 1998;13(11):2781-2798.

55. Gröne H, Warnecke E, Olbricht C. Characteristics of renal tubular atrophy in experimental renovascular hypertension: a model of kidney hibernation. Nephron. 1996;72(2):243-252.

56. Kriz W, Elger M, Hosser H, Haehnel B, Kränzlin B, Gretz N. How does podocyte damage result in tubular damage? Kidney Blood Press Res. 1999 22(1-2):26-36

57. Kriz W, et al. Pathways to recovery and loss of nephrons in anti-Thy-1 nephritis. J Am Soc Nephrol. 2003;14(7):1904-1926.
58. Hakroush S, et al. Effects of increased renal tubular vascular endothelial growth factor (VEGF) on fibrosis, cyst formation, and glomerular disease. Am J Pathol. 2009;175(5):1883-1895.

59. Fujigaki Y, et al. Transient myofibroblast differentiation of interstitial fibroblastic cells relevant to tubular dilatation in uranyl acetate-induced acute renal failure in rats. Virchows Arch. 2005; 446(2):164-176.

60. Desmouliere A, Chaponnier C, Gabbiani G. Tissue repair, contraction, and the myofibroblast. Wound Repair Regen. 2005;13(1):7-12.

61. Bulger R, Nagle R. Ultrastructure of the interstitium in the rabbit kidney. Am J Anat. 1973; 136(2):183-204.

62. Diamond J, van Goor H, Ding G, Engelmyer E. Myofibroblasts in experimental hydronephrosis. Am J Pathol. 1995;146(1):121-129.

63. Maxwell P, Ferguson D, Nicholls L, Johnson M, Ratcliffe $P$. The interstitial response to renal injury - fibroblast-like cells show phenotypic changes and have reduced potential for erythropoietin gene expression. Kidney Int. 1997;52(3):715-724.

64. Eknoyan G, McDonald MA, Appel D, Truong L. Chronic tubulo-interstitial nephritis: correlation between structural and functional findings. Kidney Int. 1990;38(4):736-743.

65. Lin SL, Kisseleva T, Brenner D, Duffield J. Pericytes and perivascular fibroblasts are the primary source of collagen-producing cells in obstructive fibrosis of the kidney. Am J Pathol. 2009;173(6):1617-1627.

66. Broekema M, et al. Bone marrow-derived myofibroblasts contribute to the renal interstitial myofibroblast population and produce procollagen I after ischemia/reperfusion in rats. J Am Soc Nephrol. 2007;18(1):165-175.

67. Li J, Deane J, Campanale N, Bertram J, Ricardo S. The contribution of bone marrow-derived cells to the development of renal interstitial fibrosis. Stem Cells. 2007;25(3):697-706.

68. Kaissling B, Hegyi I, Loffing J, Le Hir M. Morphology of interstitial cells in the healthy kidney. Anat Embryol. 1996;193(4):303-318.

69. Dawson T, Gandhi R, Le Hir M, Kaissling B. Ecto5 '-nucleotidase: localization in rat kidney by light microscopic histochemical methods. J Histochem Cytochem. 1989;37(1):39-47.

70. Wells R. The epithelial-to-mesenchymal transition in liver fibrosis: here today, gone tomorrow? Hepatology. 2010;51(3):737-740. 